\title{
A New Appoaroch in Diagnosis of Palatally Impacted Maxillary Canine in Orthodontic Patient by Cone Beam Computed Tomography (CBCT) - A Case Report
}

\author{
Dr.Rajesh Kishnani ${ }^{1}$, Dr. Rakhi Bharat ${ }^{2}$ \\ I'(Department of Orthodontics, Sri Aurobindo College of Dentistry \& PG Institute, Indore, M.P., India.) \\ ${ }_{2}^{2}$ (Department of Orthodontics, Sri Aurobindo College of Dentistry \& PG Institute, Indore, M.P., India.)
}

\begin{abstract}
In recent years cone beam computed tomography (CBCT) has become a widely accepted radiographic tool for diagnosis, treatment planning and follow-up in dentistry .3D imaging has improved diagnostic efficiency and the practice of dentistry in a variety of ways; from routine evaluation to complex analysis of unusual pathology and congenital deformities, the technology available today makes dentistry better, and easier, and more accurate.

The most recognized need for CBCT imaging in orthodontics is that of impacted canine evaluation.

In this article a patient having impacted left maxillary canine is evaluated by the $3 D C B C T$ and found beneficial particularly in terms of anatomical detail root resorption and the labiolingual relationships of the impacted tooth with the roots of neighboring teeth, in addition to determining the pathway for biomechanical resolution. Linear and angular measurements on CBCT images are accurate and precise and can be used to assess the exact position of palatal displaced canines.
\end{abstract}

Key words: $C B C T$, Impacted Canine, $C B C T$ in Orthodontics.

\section{Introduction}

Diagnosis and treatment planning of palatal displaced canines (PDCs) has until recently been based on palpation in combination with conventional two dimensional (2D) radiographs ${ }^{1}$. Since there are several disadvantages with 2D images, including distortion, inability to detect resorption of adjacent teeth, superimposition of structures, errors in projection, imaging artifacts, and variation in magnification, the application of computed tomography (CT) scanning has been suggested ${ }^{2-4}$.

Its clinical utility has been limited, however, because of the cost and the high radiation dose. 4 to address this, cone-beam computed tomography (CBCT) was introduced in the 1990s. ${ }^{5-6}$

In recent years cone beam computed tomography (CBCT) has become a widely accepted radiographic tool for diagnosis, treatment planning and follow-up in dentistry .3D imaging has improved diagnostic efficiency and the practice of dentistry in a variety of ways; from routine evaluation to complex analysis of unusual pathology and congenital deformities, the technology available today makes dentistry better, and easier, and more accurate.

$\mathrm{CBCT}^{7}$ allows the acquisition of three-dimensional volumes of the dental arches and surrounding tissues at a high spatial resolution and a low radiation dose. Three dimensional imaging is the volumetric imaging which gives the information in depth length and width. CBCT is a compact faster and safer version of regular CT it provides high-resolution imaging, diagnostic reliability, and risk-benefit assessment. It is an accurate tool for many clinical oral-maxillofacial indications, with lower radiation doses than computerized tomography.

For orthodontics, one single CBCT scan can effectively generate all the images needed for orthodontic diagnosis including the lateral cephalograph, the panoramic radiograph, the antero-posterior cephalogram, temporo-mandibular joint tomograms, and many other oblique/cross-sectional slices previously unavailable in flat planar films at a relatively equivalent radiation dose, for a set of orthodontic X-ray initial records.

Possibly the most recognized need for CBCT imaging in orthodontics is that of impacted canine evaluation. . The prevalence of impacted maxillary canines is approximately $0.9 \%$ to $3.0 \%$. The ratio of palatal to labial impactions has been shown to be as high as 9:1.

In the past, orthodontists have used the tube shift technique to compare two periapical radiographs taken at different beam angles to determine the facial/lingual position of the impacted canine. This same lingual, opposite buccal rule is helpful in determining whether the impacted canine is labial or lingual to the incisor roots; however, the degree of displacement is difficult to determine. CBCT imaging is precise in determining not only the labial/lingual relationship but also more exact angulations of the impacted canine. These 3D images are beneficial in determining the proximity of adjacent incisor and premolar roots, which can be invaluable in 
determining the ease of uncovering and bonding and the vector of force that should be used to move the tooth into the arch with a lesser chance of adjacent root resorption.

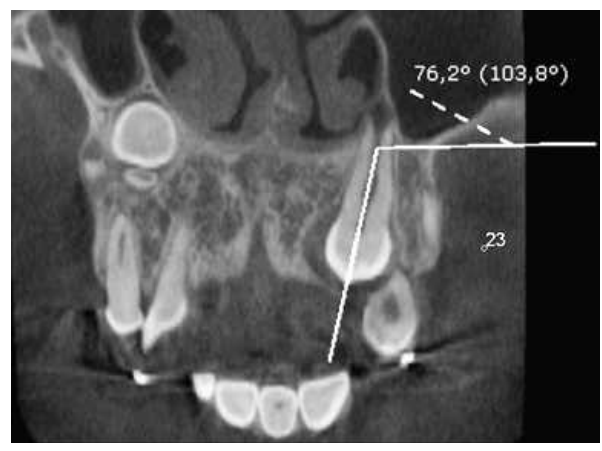

Mesioangular angle of PDC measured on a coronal view

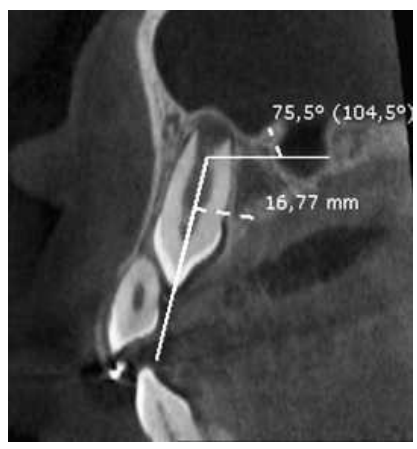

Figure: 1

sagital angle and vertical position measured on a sagital view

\section{Case report}

A $20 \mathrm{yr}$ old female patient came to the department of orthodontic and Dentofacial orthopedic in SAIMS College of dentistry Indore with the chief complained of forward placement of upper and lower frontal teeth.

\section{Figure: 2 Extra-Oral Photographs}
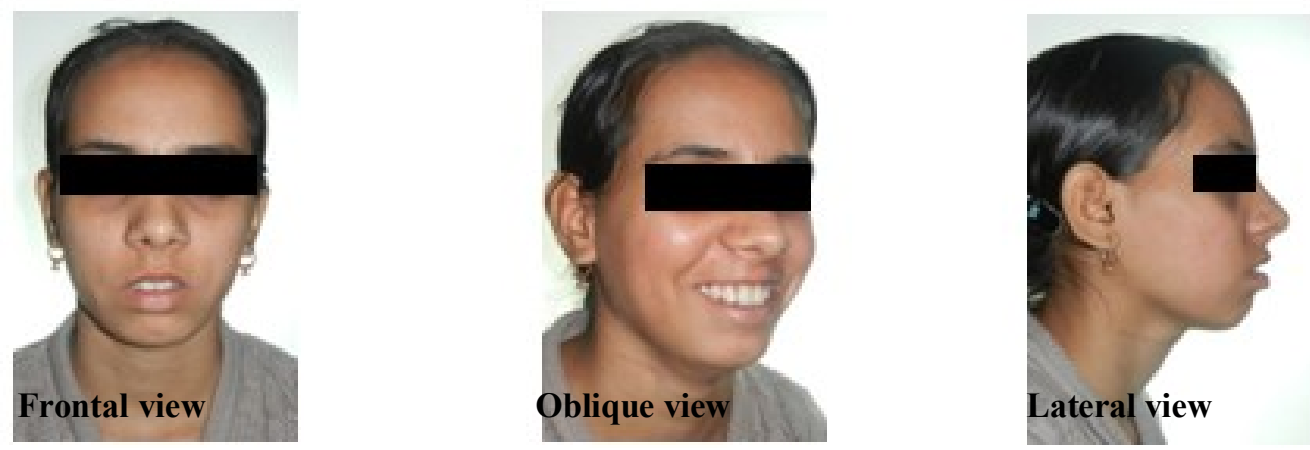

\section{Extra-oral findings}

The Extraoral clinical examination demonstrated she had a convex profile, protrusive upper lip, lip incompetence and shallow mentolabial sulcus (Fig-1)

Figure: 3 Intra-Oral Photographs

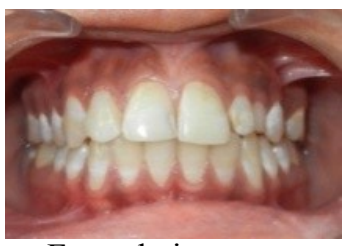

Frontal view

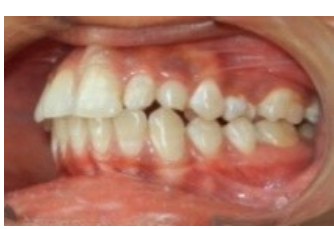

Left lateral view

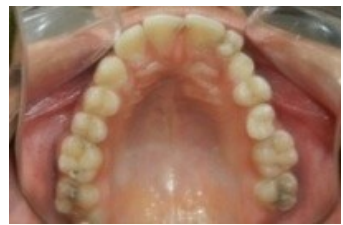

Maxillary occlusal view

\section{Intra-oral findings}

The intraoral clinical examination revealed that the molar relationship on the right side is Angle Class II, and end on left side due to the rotation of maxillary $2^{\text {nd }}$ premolar.

Retained maxillary deciduous canine also present on left side, Bimaxillary protrusion. The overjet and overbite is $4 \mathrm{~mm}$. Both of the upper and lower dental arch form are ovoid and symmetrical in shape mid line of both arches are coinciding (Fig-3) 
Radiographic findings

Occlusal and IOPA $\mathrm{x}$ - ray shows impacted left maxillary canine

The panoramic radiograph showed the absence of all the third molars, root canal treated mandibular $2^{\text {nd }}$ premolar on both side and impacted maxillary canine on left side.

The cephalometric findings revealed a skeletal Class II jaw relation, increased ANB angle,

The soft tissue analysis showed an acute naso-labial angle and protrusive upper and lower lips.

Figure: 4 Routine radiographs (giving 2-D- image) for diagnosis of impacted canine

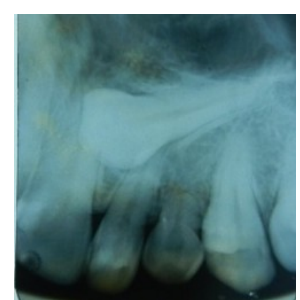

Periapical radiograph

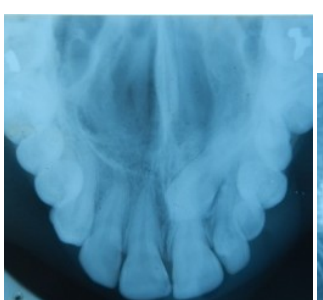

occlusal radiograph

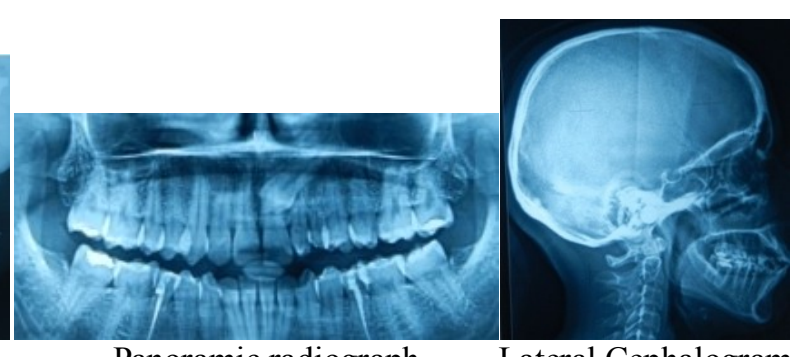

Panoramic radiograph
Lateral Cephalogram,

For CBCT imaging of impacted canine in this patient KODAK-CS-9300 CBCT MACHINE has been used. CBCT scans are far better than conventional panoramic radiographs in verifying the orientation and location of the impacted canine and its relationship to neighboring structures. This technique makes identification of the exact position and shape of impacted canines possible, which is crucial in treatment planning. Furthermore, it is very helpful in evaluating damage to adjacent teeth and the amount of surrounding bone.
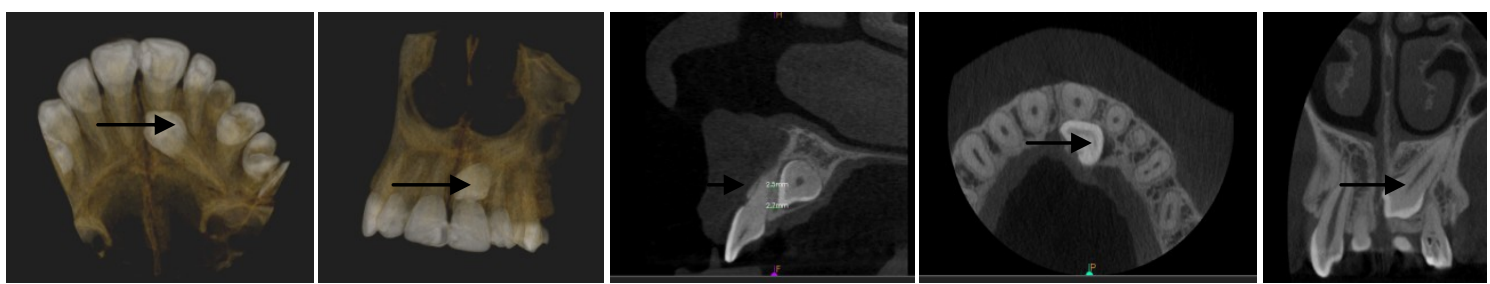

Figure: 5 CBCT images showing the palatal position of tooth 23. the relationship between teeth 22. , Sagital CBCT image displaying the proximity of 23 to the root of 22.

\section{Diagnosis}

The patient was diagnosed with a skeletal Class II pattern and dental Class -I on right side end on left side with bimaxillary protrusion and vertical grower.

\section{Treatment objectives}

The treatment objectives are to (1) Achieve Class I molar on both side (2) Correction of bimaxillary protrusion (3) Alignment of left permanent canine in upper arch

(2) Improve the lip posture

\section{Treatment plan}

In-Orthodontic treatment after alignment and leveling of the upper and lower arches, surgical exposure of palatally impacted canine has been done. And begg's bracket bonded with ligature tie, this ligature tie helps the canine for retraction and exposure in oral cavity... when canine exposed, Kalra's K-9 spring8 attached to canine for proper alignment in arch.

Figure: 6 surgical procedures for exposure of impacted canine

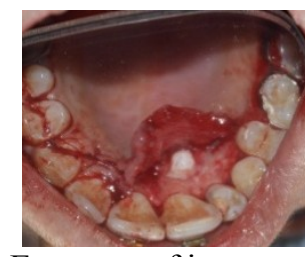

Exposure of impacted Canine

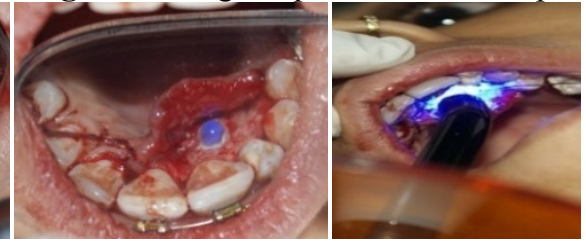

bonding of bracket

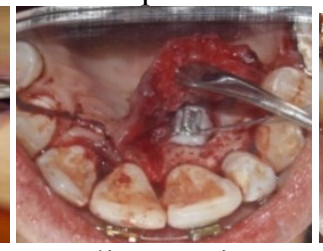

ligature tie

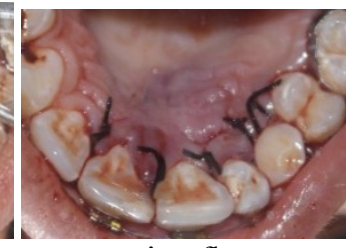

suturing flap 


\section{Conclusion}

CBCT in relation to the treatment of impacted teeth are more beneficial particularly in terms of anatomical detail ${ }^{9}$ root resorption and the labiolingual relationships of the impacted tooth with the roots of neighboring teeth, in addition to determining the pathway for biomechanical resolution. Linear and angular measurements on CBCT images are accurate and precise and can be used to assess the exact position of palatal displaced canines. ${ }^{10}$

\section{References}

[1]. Mason C, Papadakou P, Roberts GJ. The radiographic localization of impacted maxillary canines: a comparison of methods. Eur J Orthod. 2001; 23:25-34.

[2]. Elefteriadis JN, Athanasiou AE. Evaluation of impacted canines by means of computerized tomography. Int J Adult Orthodon Orthognath Surg. 1996; 11:257-264.

[3]. Bodner L, Bar-Ziv J, Becker A. Image accuracy of plain film radiography and computerized tomography in assessing morphological abnormality of impacted teeth. Am J Orthod Dentofacial Orthop. 2001; 120:623-628.

[4]. Preda L, La Fianza A, Di Maggio EM, Dore R, Schifino MR, Campani R. The use of spiral computed tomography in the localization of impacted maxillary canines. DentomaxillofacRadiol. 1997; 26:236-241.

[5]. Kau CH, Richmond S, Palomo JM, Hans MG. Threedimensional cone beam computerized tomography in orthodontics. J Orthod. $2005 ; 32: 282-293$.

[6]. Ludlow JB, Davies-Ludlow LE, Brooks SL, Howerton WB.Dosimetry of 3 CBCT devices for oral and maxillofacial radiology: CBMercuRay, NewTom 3G and i-CAT. Dentomaxillofac Radiol. 2006; 35:219-226

[7]. Ruben pauweis et al. Effective dose range for dental cone beam computed tomography. European Journal of Radiology 2010 ; $1-5$.

[8]. Varun kalra, the k-9 spring for alignment of impacted canine. Jco/October 2000, 34; 606-609

[9]. William E. Harrell, Jr. 3D Diagnosis and Treatment Planning in Orthodontics .Semin Orthod 2009;15:35-41

[10]. Julia Naoumovaa; Heidrun Kjellbergb; Reet Palmc Cone-beam computed tomography for assessment ofpalatal displaced canine positionA methodological study-Angle Orthodontist, 2014, 84; 459-466 